\title{
Geographical distribution of hypertensive disorders in pregnancy and their adverse maternal and perinatal outcomes in Thailand
}

\begin{abstract}
Hypertensive disorders in pregnancy are a public health concern which has important adverse impacts on maternal and perinatal health, especially in developing countries. Among the hypertensive disorders in pregnancy, severe preeclampsia and eclampsia are contributed to the major causes of maternal and perinatal mortality and morbidity. Hypertensive disorders in pregnancy are found to be varied among different ethnicity and socio-economic status. This study aimed to assess the geographical distribution of hypertensive disorders in pregnancy across provinces of Thailand and the adverse maternal and perinatal outcomes. A secondary analysis of the hospital-based data retrieved from the 2014 database of the Thailand National Health Security Office (NHSO) using the 10th revision of the International Statistical Classification of Diseases and Related Health Problems (ICD) for pregnancy, childbirth and postpartum conditions. All admitted pregnant women aged 10-49 years with ICD-10 for hypertensive disorders in pregnancy were analyzed. A total of 315,126 women delivered, the incidence of hypertensive disorders in pregnancy in Thailand was 27.5 per 1,000 deliveries. The incidence of severe preeclampsia/eclampsia in women aged 335 years was double or triple compared to in those aged $<20$ or 20 -34years and more common in central, southern and eastern areas than in northern and northeastern areas. Among women with severe preeclampsia/eclampsia, $65.8 \%$ of them underwent cesarean section, $16.5 \%$ had preterm delivery and $3.4 \%$ presented postpartum hemorrhage. The findings of this study will guide the health personnel and policy makers to make the well-planned strategies for health system and services in accordance with the incidence of hypertensive disorders in pregnancy by geographical variation and their adverse maternal and perinatal health.
\end{abstract}

Keywords: hypertensive disorders in pregnancy, severe preeclampsia, eclampsia, adverse maternal and perinatal outcomes
Volume 2 Issue 2 - 2017

\author{
Tippawan Liabsuetrakul,' Thida² \\ 'Department Epidemiology Unit, Prince of Songkla University, \\ Thailand \\ ${ }^{2}$ Department of Medical Research, Ministry of Health and \\ Sports, Myanmar
}

Correspondence: Tippawan Liabsuetrakul, Epidemiology Unit, Faculty of Medicine, Prince of Songkla University, Hat Yai, Songkhla, Thailand, Tel +66-74-45 I I65; Fax +66-74-429754, Email Itippawa@hotmail.com

Received: January 30, 2017 | Published: April 10, 2017
Abbreviations: NHSO, national health security office; ICD, international statistical classification of diseases

\section{Introduction}

Hypertensive disorders in pregnancy is common global obstetric concerns which affect to the occurrence of adverse maternal and perinatal health, especially in all countries. ${ }^{1}$ Severe preeclampsia and eclampsia are the major causes of adverse maternal and perinatal mortality and morbidity worldwide including in Thailand. ${ }^{1,2}$ Since the etiology of hypertensive disorders in pregnancy is not well-known and unpredictable, the recognition of its geographic distribution is essential for the health personnel and policy makers in order to prioritize interventions, resource allocation for providing optimal health services to the high risk pregnant women. However, there were no the study using the national data for geographical distribution of hypertensive disorders in pregnancy in Thailand. In addition, the previous studies of the adverse maternal and perinatal outcomes in hypertensive disorders in pregnancy may be limited due to smaller sample size. ${ }^{3-5}$ Therefore, this study aimed to assess the geographical distribution of hypertensive disorders in pregnancy across provinces of Thailand and adverse maternal and perinatal outcomes in different subgroups of hypertensive disorders in pregnancy.

\section{Methods}

A secondary analysis of the hospital-based data retrieved from the 2014 database of the Thailand National Health Security Office (NHSO) using the 10th revision of the International Statistical Classification of Diseases and Related Health Problems (ICD) for pregnancy, childbirth and postpartum conditions (O00-O99). All admitted pregnant women aged 10-49 years who delivered in the study period were analyzed.

The ICD-10 for hypertensive disorders in pregnancy using the code categories as $\mathrm{O} 10, \mathrm{O} 11, \mathrm{O} 13, \mathrm{O} 14, \mathrm{O} 15$ and $\mathrm{O} 16$ of which were classified into two groups:

a. Severe preeclampsia/eclampsia using O14.1 (severe preeclampsia), O14.2 (HELLP syndrome), O14.9 (preeclampsia, unspecified), O15.0 (eclampsia in pregnancy), O15. 1 (eclampsia in labor), O15.2 (eclampsia in the puerperium), and O15.9 (eclampsia, unspecified as to time period).

b. Other types of hypertension using the rest of hypertensive disorders in pregnancy. Common maternal and perinatal outcomes measured included the postpartum hemorrhage, disseminated intravascular coagulation, renal failure, cesarean section, preterm delivery and stillbirth. The data were managed and analy- 
zed using R software version 3.1.3 (R Foundation for Statistical Computing 2015, Vienna, Austria).

The geographical distribution of hypertensive disorders in pregnancy across provinces of Thailand and adverse maternal and perinatal outcomes in different groups of hypertension were presented descriptively. This analysis was approved by the Institute Ethics Committee of Faculty of Medicine, Prince of Songkla University, Thailand.

\section{Results}

Of 315,126 women delivered, the incidence of hypertensive disorders in pregnancy in Thailand was 27.5per 1,000 deliveries of which 10.1 was severe preeclampsia/eclampsia and 17.4 was other types of hypertension. The incidence of severe preeclampsia/ eclampsia in women aged $\geq 35$ years was double or triple compared to in those aged $<20$ or 20-34years and more common in central, southern and eastern areas than in northern and northeastern areas (Table 1). Rates of cesarean section among women with severe preeclampsia/eclampsia and other types of hypertension were $65.8 \%$ and $50.6 \%$, respectively. Preterm delivery was found in those with severe preeclampsia/eclampsia for $16.5 \%$ and with other types of hypertension for $5.7 \%$. Postpartum hemorrhage was seen in $3.4 \%$ in both groups.

Table I Age-specific incidence of severe preeclampsia/ eclampsia across regions of the Thailand

\begin{tabular}{lllll}
\hline \multirow{2}{*}{ Regions } & \multicolumn{4}{l}{ Age-specific incidence of severe preeclampsia/ } \\
& $<20$ years & $\mathbf{2 0 - 3 4}$ years & $\geq \mathbf{3}$ years & Total \\
\hline Central & 10.4 & 12 & 27.7 & 13 \\
Southern & 8.6 & 10.5 & 29.5 & 12.7 \\
Eastern & 6.7 & 10.1 & 22.8 & 10 \\
Northern & 5.3 & 6.6 & 21.7 & 7.6 \\
Northeastern & 5.9 & 7.1 & 14.8 & 7.5 \\
Total & $\mathbf{7 . 5}$ & $\mathbf{9}$ & $\mathbf{2 2 . 7}$ & $\mathbf{1 0 . 1}$ \\
\hline
\end{tabular}

\section{Discussion}

The incidence of hypertensive disorders in pregnancy in our study (27.5 per 1000 deliveries) was similar to the finding of secondary analysis of the World Health Organization Multicounty Survey on Maternal and Newborn Health from 29 countries in Africa, Asia, Latin America and the Middle East (27.3 per 1000 deliveries) and showed various geographic distribution. ${ }^{6}$ A previous study conducted in central of Thailand reported the prevalence of preeclampsia accounted for 47 per 1000 deliveries. $^{7}$ The different reports on the incidence of hypertensive subgroups was mainly because the different classifications were chosen..$^{5-7}$ Maternal age at $\geq 35$ years were consistently reported to be the concurrent risk of hypertensive disorders in pregnancy. ${ }^{5,8,9}$ Maternal near-miss was increased among women with hypertensive disorders. ${ }^{1}$ Higher cesarean section was higher in China. ${ }^{9}$ Preterm birth and stillbirth increased in women with hypertensive disorders same as in a study in China, ${ }^{9} \mathrm{Africa}^{10}$ and The United States. ${ }^{11}$ The findings of this study confirmed the importance of hypertensive disorders in pregnancy in which the policy action is required.

\section{Conclusion}

Hypertensive disorders in pregnancy in Thailand had a different geographic distribution. Pregnant women with advanced maternal age had an increased risk of the occurrence of severe preeclampsia/ eclampsia. Common adverse maternal and perinatal outcomes were preterm delivery and postpartum hemorrhage. Early detection and prevention of adverse maternal and perinatal outcomes in women with hypertensive disorders are essential.

\section{Acknowledgements}

None.

\section{Conflict of interest}

Author declares that there is no conflict of interest.

\section{References}

1. Carmen D, Carla A. Global burden of hypertensive disorders of pregnancy in the year 2000. Geneva: WHO; 2003.

2. Khan KS, Wojdyla D, Say L, et al. WHO analysis of causes of maternal death: a systematic review. Lancet. 2006;367(9516):1066-1074.

3. Olusanya BO, Solanke OA. Perinatal outcomes associated with maternal hypertensive disorders of pregnancy in a developing country. Hypertens Pregnancy. 2012;31(1):120-130.

4. Buga GA, Lumu SB. Hypertensive disorders of pregnancy at Umtata General Hospital: perinatal and maternal outcomes. East Afr Med J. 1999;76(4):217-222.

5. Lisonkova S, Joseph KS. Incidence of preeclampsia: risk factors and outcomes associated with early-versus late-onset disease. Am J Obstet Gynecol. 2013;209(6):544.e1-544.e12.

6. Abalos E, Cuesta C, Carroli G, et al. Pre-eclampsia, eclampsia and adverse maternal and perinatal outcomes: a secondary analysis of the World Health Organization Multi-country Survey on Maternal and Newborn Health. BJOG. 2014;121(Suppl 1):14-24.

7. Pitakkarnkul S, Phaloprakarn C, Wiriyasirivaj B, et al. Seasonal variation in the prevalence of preeclampsia. J Med Assoc Thai. 2011;94(11):1293-1298.

8. Luealon P, Phupong V. Risk factors of preeclampsia in Thai women. $J$ Med Assoc Thai. 2010;93(6):661-666.

9. Zhu YC, Yang HX, Wei YM, et al. Analysis of correlation factors and pregnancy outcomes of hypertensive disorders of pregnancy-a secondary analysis of a random sampling in Beijing, China. J Matern Fetal Neonatal Med. 2016;30(6):751-754.

10. Johnson KM, Zash R, Haviland MJ, et al. Hypertensive disease in pregnancy in Botswana: Prevalence and impact on perinatal outcomes. Pregnancy Hypertens. 2016;6(4):418-422.

11. Gibbins KJ, Silver RM, Pinar H, et al. Stillbirth, hypertensive disorders of pregnancy, and placental pathology. Placenta. 2016;43:61-68. 